\title{
Association Between APOLI Genotype and Kidney Diseases and Annual Kidney Function Change: A Systematic Review and Meta-Analysis of the Prospective Studies
}

This article was published in the following Dove Press journal:

International Journal of Nephrology and Renovascular Disease

\section{Ram Jagannathan (iD) \\ Kanya Rajagopalan ${ }^{2}$ \\ Julien Hogan ${ }^{3,4}$ \\ Allyson $\mathrm{Hart}^{5,6}$ \\ Kenneth A Newell ${ }^{4}$ \\ Stephen O Pastan 7 \\ Rachel E Patzer ${ }^{4,7,8}$}

'Department of Medicine, Division of Hospital Medicine, Emory University School of Medicine, Atlanta, GA, USA;

${ }^{2}$ Department of Global Health, Rollins School of Public Health, Emory

University, Atlanta, GA, USA; ${ }^{3}$ Pediatric Nephrology Department, Robert Debre University Hospital, Paris, 75019, France; ${ }^{4}$ Department of Surgery, Division of Transplantation, Emory University School of Medicine, Atlanta, GA, USA; ${ }^{5}$ Division of Nephrology, Department of Medicine, Hennepin Healthcare, Minneapolis, MN, USA; 'University of Minnesota Medical School, Minneapolis, MN, USA; ${ }^{7}$ Department of Medicine, Emory University School of Medicine, Atlanta, GA, USA; ${ }^{8}$ Department of Epidemiology, Rollins School of Public Health, Emory University, Atlanta, GA, USA
Correspondence: Ram Jagannathan Department of Medicine, Division of Hospital Medicine, Emory University School of Medicine, Atlanta, GA, 30322, USA

Email ram.jagannathan@emory.edu
Background: Two coding risk variants in the Apo L1 gene (APOL1) underlie most of the excess risk for kidney diseases in recent African ancestry patients. Strength and consistency of the relationship between APOL1 high-risk genotypes and the risk of chronic kidney diseases (CKD) and end-stage renal disease (ESRD) are not uniform.

Objective: To conduct a systematic review and meta-analysis of prospective studies assessing the association of APOL1 genotypes and the risk of developing CKD, ESRD, and CKD to ESRD in adults.

Methods: Systematic search of MEDLINE, EMBASE, and Google Scholar was performed for prospective studies assessing the associations between APOL1 genotypes and CKD, ESRD, and progression from CKD to ESRD. Secondary analyses were to evaluate the annual kidney function change by APOL1 gene status. Random effects models were used to estimate pooled risk ratios (RRs) and weighted mean differences for outcomes of interest. Results: The search yield 10 prospective during a follow-up period ranging from 4.4 to 25 years. The high-risk APOL1 genotype was associated with the incidence of CKD (RR:1.41 [95\% CI: 1.14-1.75]), the progression from CKD to ESRD (RR: 1.70 [95\% CI:1.44; 2.01]) compared with the low-risk APOL1 genotype. There was no appreciable association between high-risk APOL1 genotype with the incidence of ESRD. Furthermore, high-risk APOL1 genotype was associated with a marginal decrement in the annual eGFR decline $(-0.55[95 \%$ CI: -0.94 to -0.16$]) \mathrm{mL} / \mathrm{min} / 1.73 \mathrm{~m}^{2}$ compared with low-risk APOL1 genotype status.

Conclusion: In summary, African Americans carrying APOL1 high-risk genotypes are at increased risk of developing CKD and ESRD. Given that the APOL1 risk alleles are common among individuals with African ancestry, with $\sim 18 \%$ of African Americans carrying high-risk alleles, these findings highlight the potential identification of subgroups of patients who may benefit from APOL1 screening and developing culturally-appropriate interventions.

Keywords: APOL1, chronic kidney disease, end-stage renal diseases, disparities, African Americans

\section{Introduction}

Significant disparities exist in kidney disease, with African Americans facing an increased burden of chronic kidney diseases (CKD) and progression from CKD to end-stage renal diseases (ESRD). ${ }^{1}$ Besides, African Americans experience rapid kidney function deterioration at the early stages of the disease, compared with 
Caucasians. ${ }^{2}$ The increased risk is partly attributable to two kidney risk variants (APOL1 G1 and G2) in the gene encoding apo L1 (APOL1). ${ }^{3}$ Unlike the other common complex disease variants that follow additive or multiplicative patterns, APOL1-mediated kidney disease risk principally carries autosomal-recessive inheritance. ${ }^{3}$ Although the literature suggests an association between high-risk APOL1 genotypes and kidney diseases, the strength of association was not uniform, with some studies finding a strong association of high-risk APOL1 status with kidney diseases and other studies finding weaker associations. Although a few reports and reviews were available on the association of APOL1 risk variants from case-control studies, ${ }^{4,5}$ no systematic review or metaanalysis has evaluated the association between APOL1 risk status and the incidence of CKD, ESRD, and the progression of CKD to ESRD, and kidney function decline. This is important as there is an ongoing debate on the usefulness of APOL1 genotyping in clinical decision making, with only a little consensus, has emerged among clinicians. ${ }^{6,7}$

Therefore, we conducted a systematic review and a meta-analysis of prospective cohort studies to describe the association between APOL1 genotypes and future risk of CKD, ESRD, and progression from CKD to ESRD.

\section{Methods}

\section{Selection Strategy and Inclusion Criteria}

We used a predefined search strategy and statistical analysis plan for this systematic review and meta-analysis using a prespecified protocol. Using the Meta-analysis Of Observational Studies in Epidemiology (MOOSE) guidelines for reporting, ${ }^{8}$ we searched electronic databases (MEDLINE, Google Scholar, and Embase) for prospective cohort studies up to 31 March 2020, utilizing a systematic search strategy that removed duplicate studies. A combination of free text words and Medical Subject Headings (MeSH) terminologies were employed to search, and multiple pre-searches determined the final search strategy without language restrictions. We have used the following terms for the MEDLINE:

((('renal insufficiency, chronic'[MeSH Terms] OR 'kidney failure, chronic' [MeSH Terms]) OR 'proteinuria' $[\mathrm{MeSH}$ Terms]) OR 'albuminuria' [MeSH Terms]) OR (chronic kidney disease[Text Word] OR chronic kidney failure [Text Word]) OR chronic kidney insufficiency[Text Word] OR chronic kidney dysfunction[Text Word] OR chronic renal disease[Text Word] OR chronic renal failure[Text Word] OR chronic renal insufficiency[Text Word] OR chronic renal dysfunction[Text Word] OR end stage renal disease[Text Word] OR proteinuria[Text Word] OR albuminuria[Text Word] AND (('apolipoprotein 11' [MeSH Terms] OR ('apolipoprotein'[All Fields] AND '11'[All Fields]) OR 'apolipoprotein 11'[All Fields]) AND ('humans'[MeSH Terms] OR 'humans'[All Fields] OR 'human'[All Fields]) OR APOL1[Text Word])

To identify eligible studies. Analogous search terms were used for the other electronic databases. Finally, we manually searched for eligible original research, reviews, and related articles to compensate for the electronic search deficiency. If multiple studies reported the same cohort results, the latest and most complete data was extracted for the analysis.

\section{Study Selection}

Studies were included in this meta-analysis if they fulfilled the following selection criteria: a) prospective study design; b) measured APOL1 gene alleles; c) the outcome was CKD/ESRD; d) published in English; e) sample size of $>100$ subjects; and $f$ ) the investigators reported absolute counts for calculating the risk ratio (RR) with $95 \% \mathrm{CI}$ for the outcomes of interest. We excluded any animal, case-control, cross-sectional studies, reviews, commentaries, and letters that examined other associations. If the study results were published more than once, we included the finding with the complete follow-up in our primary analysis. Two reviewers (JR, RK) independently and manually screened the abstracts using the computerized screening program Abstrackr (Tufts Medical Center, Boston, Massachusetts). ${ }^{9}$ Conflicts over inclusion were resolved with an in-person discussion with a third investigator (RP) if necessary.

\section{Data Extraction}

Data were extracted and captured into a spreadsheet specifically for this study with a data validation feature to control the data (type, value, and find invalid entries) entered. Variables entered from the selected studies included: a) study-level information (authors, country, center, year); b) study population characteristics (age, sex, and follow-up duration); and c) the number of events (CKD/ESRD). Two reviewers independently extracted data. Discrepancies were resolved by consensus or by an adjudicator (RP) if needed. 


\section{Quality Assessment}

One author (JR) assessed the methodological quality. Since the included studies (except one) were nonrandomized and had a cohort design, Newcastle-Ottawa Quality Assessment Scale (NOS) ${ }^{10}$ was used to judge study quality, as advocated by the Cochrane collaboration. ${ }^{11}$ NOS uses a star system (with a maximum of nine stars) to appraise a study in three domains: the selection of participants (4 points), comparability of study groups ( 2 points), and the ascertainment of outcomes of interest ( 3 points). We assigned $0-3,4-6$, and 7-9 for the low, moderate, and high quality of studies, respectively.

\section{Data Synthesis and Statistical Analysis} Primary Exposure Variable

The primary exposure was the existence of high-risk APOL1 genotypes, using a recessive model (G1/G1, G2/G2, or G1/ $\mathrm{G} 2$ ) compared with low-risk genotypes (G1/G0, G2/G0, or G0/G0). The G1 haplotype consists of two nonsynonymous missense variants (rs73885319 [S342G], and rs60910145 [I384M]) that are in almost total positive linkage disequilibrium, and the $\mathrm{G} 2$ haplotype was characterized by a six-base pair in-frame deletion (rs71785313 [N388del: Y389del]). These alleles are in negative disequilibrium and virtually never occur on the same chromosome. ${ }^{12}$

\section{Outcome Variables}

The primary outcomes were the incidence of CKD, ESRD, and the progression from CKD to ESRD using an autosomal recessive genetic model (Supplemental Table 1).

\section{Statistical Analysis}

In this meta-analysis, the relative risks (RR) and 95\% confidence intervals were appraised as the effect size for all the studies, and hazard ratios (HR) were deemed equivalent to $\mathrm{RR}^{13}$ If outcomes were presented as odds ratios (ORs), ${ }^{14}$ data were converted to $R R$ for analysis by using the formula $\mathrm{RR}=\mathrm{OR} /([1-\mathrm{pRef}]+[\mathrm{pRef} \times \mathrm{OR}])$, where $\mathrm{pRef}$ is the prevalence of the outcome (ie, kidney diseases) in the reference group. ${ }^{15}$ Outcomes from individual cohort studies were pooled using a random-effects model because this approach assumes that there could be heterogeneity in clinical and methodological approaches that might affect the findings. All pooled analyses were reported with $95 \%$ CIs. The DerSimonian and Laird method of moments estimator were used to estimate the between-study variance, and the corresponding 95\% CIs were calculated using the Wald type method. ${ }^{16}$ Secondary analyses were defined a priori to evaluate the annual kidney function change by APOL1 gene status. As the eGFR measurements were based on different methodologies, we reported the standardized mean difference using the inverse-variance method. ${ }^{17} \mathrm{~A} \mathrm{P}$ value $<0.05$ was regarded as statistically significant. We assessed heterogeneity from the Mantel-Haenszel model and $\mathrm{I}^{2}$ values (the percentage of variance in the pooled estimate due to between-study differences), with an $\mathrm{I}^{2}$ value greater than $50 \%$, suggesting moderate heterogeneity. ${ }^{18}$ The possibility of publication bias was evaluated for the overall analysis using the Begg test and visual inspection of a funnel plot. ${ }^{19}$ Additional sensitivity analyses were undertaken by omitting the most extensive study or studies based on their weighted percentage in the pooled estimates. ${ }^{20}$ All meta-analyses were conducted in $\mathrm{R}$ (version 4.0.1.; $\mathrm{R}$ foundation of statistical computing, Vienna, Austria) statistical platform using the packages "meta" (version 4.9-6) (12) and "metafor" (version 2.1-0) (13), with a two-tailed $\alpha$ of 0.05 considered statistically significant.

\section{Results Characteristics and Quality of the Included Studies}

Among 199 candidate abstracts identified in the electronic databases and other sources, we identified 30 potential studies for a full review. Twenty studies were excluded on full manuscript assessment as they deemed non-relevant (Supplemental Figure 1). A detailed review and data extraction was conducted on ten studies ${ }^{14,21-29}$ for systematic review and meta-analysis (Supplemental Figure 1). All included studies were based in North America. There were 53,976 (high-risk APOL1 genotype: 7376; low-risk APOL1 genotype: 46,600) participants across the ten cohort studies (Table 1). The prevalence of the APOL1 kidney risk genotype was $17.5 \%$, ranging from $12.2 \%$ in the CARDIA study, a cohort of younger adults, to $26.6 \%$ in the JHS, a large, community-based, observational study. The age range was 34.9 years to 61.7 years, with a median age of 54.1 years. The proportion of females included in the study was $48.0 \%$ (range: $0 \%-100 \%$ ); and median baseline eGFR was 92.5 (non-CKD: 107.4; CKD: 61.2 ) $\mathrm{mL} / \mathrm{min} / 1.73 \mathrm{~m}^{2}$. The duration of follow-up ranged from 4.4 to 25 years, with a median duration of 10.0 years. All but two studies genotyped APOL1 G1 (rs73885319 and rs60910145) and G2 (rs71785313) using the Taqman assay (Thermo Fisher Scientific). According to quality assessment criteria, seven 


\begin{tabular}{|c|c|c|c|c|c|c|c|c|c|c|c|c|}
\hline 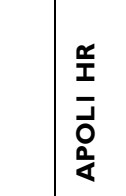 & 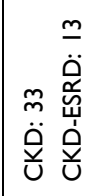 & $\begin{array}{l}\text { ה } \\
\ddot{\theta} \\
\text { U }\end{array}$ & $\begin{array}{l}n \\
o \\
\ddot{0} \\
\text { 岀 } \\
\text { 仓े } \\
\text { ü }\end{array}$ & 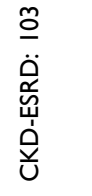 & 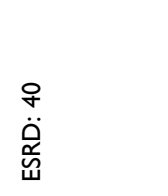 & $\stackrel{\alpha}{z}$ & 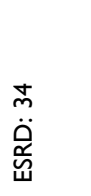 & $\frac{\alpha}{z}$ & $\begin{array}{l}\underline{\tilde{o}} \\
\ddot{\hat{u}}\end{array}$ & 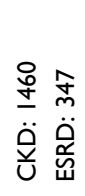 & 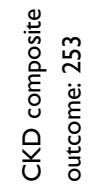 & 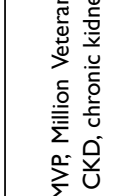 \\
\hline 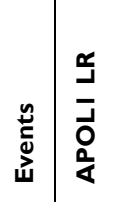 & 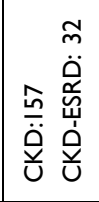 & $\begin{array}{l}\stackrel{\infty}{1} \\
\ddot{\ddot{\theta}}\end{array}$ & 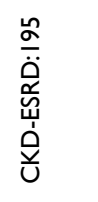 & 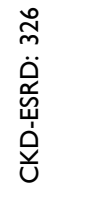 & $\begin{array}{l}\frac{\sigma}{\sigma} \\
\ddot{\ddot{o}} \\
\text { 崫 }\end{array}$ & $\stackrel{\alpha}{z}$ & 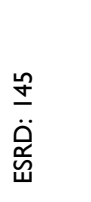 & $\frac{n}{z}$ & $\begin{array}{l}\bar{\sigma} \\
\ddot{\ddot{u}}\end{array}$ & 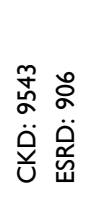 & 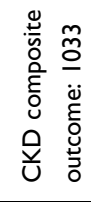 & 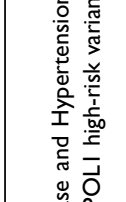 \\
\hline 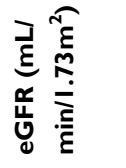 & $\bar{\Xi}$ & $\stackrel{\underline{\mathrm{I}}}{\underline{y}}$ & 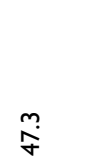 & $\stackrel{\dot{\gamma}}{\dot{\gamma}}$ & $\stackrel{\text { ñ }}{\Omega}$ & $\underset{\infty}{\infty}$ & $\bar{\Xi}$ & 品 & 兴 & $\underset{\infty}{\stackrel{\stackrel{n}{\infty}}{\infty}}$ & $\frac{\dot{0}}{\infty}$ & 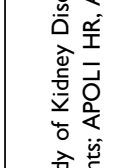 \\
\hline $\begin{array}{ll}0 \\
0 \\
\text { zo }\end{array}$ & $\infty$ & r & $\sigma$ & $\sigma$ & 1 & in & $\infty$ & $\sigma$ & r & $\sigma$ & in & 预 \\
\hline 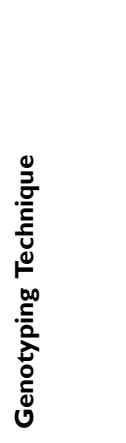 & 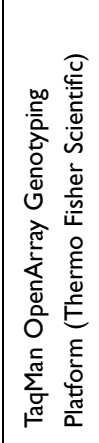 & 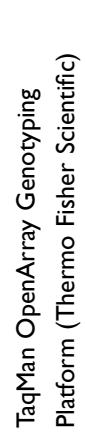 & 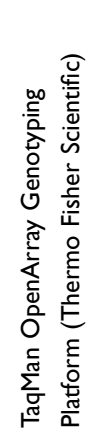 & 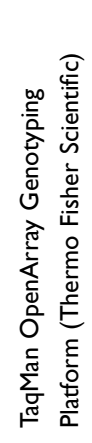 & 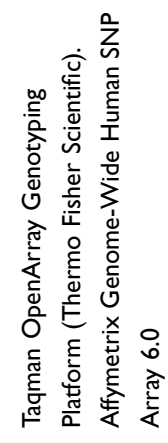 & 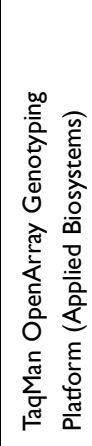 & 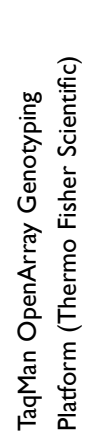 & 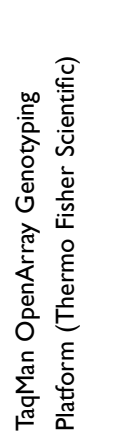 & 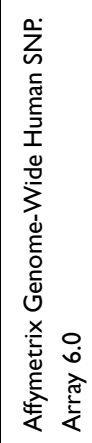 & 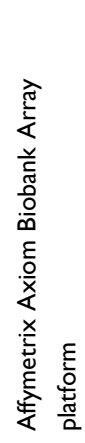 & 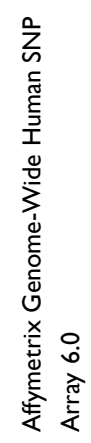 & 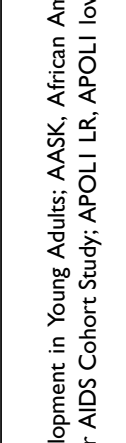 \\
\hline 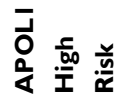 & 芠 & $\underline{\underline{I}}$ & 음 & $\stackrel{?}{\text { ㄱ }}$ & 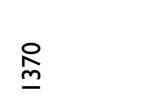 & 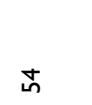 & $\stackrel{?}{f}$ & $\infty$ & $\underset{\sim}{ \pm}$ & 志 & $\hat{\kappa}$ & 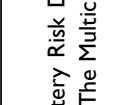 \\
\hline 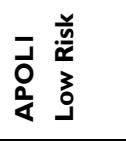 & 苂 & $\stackrel{\stackrel{亡}{\mathscr{H}}}{=}$ & $\stackrel{\tilde{m}}{n}$ & $\underline{\underline{\sigma}}$ & ลั & $\stackrel{\curvearrowright}{\text { s }}$ & ટ્లి & $\stackrel{\infty}{\mathrm{m}}$ & 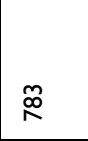 & $\begin{array}{l}\text { ปू } \\
\text { సे }\end{array}$ & $\frac{n}{2}$ & 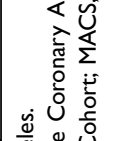 \\
\hline ङ & ठ্ল & $\stackrel{\underline{I}}{\text { I }}$ & ô & $\overline{\bar{\sigma}}$ & $\stackrel{\hat{m}}{=}$ & m & $\begin{array}{l}\text { م. } \\
\text { in }\end{array}$ & $\stackrel{8}{\stackrel{8}{\sim}}$ & 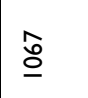 & $\begin{array}{l}\text { O. } \\
\text { ò } \\
\text { m. }\end{array}$ & 灾 & 4 \\
\hline 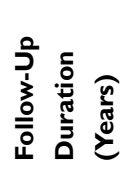 & 으 & 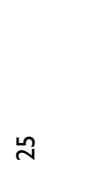 & $a$ & $\stackrel{\sim}{*}$ & $=$ & $\stackrel{\circ}{\sim}$ & 崩 & 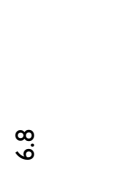 & $\stackrel{\circ}{\circ}$ & $\simeq$ & $\stackrel{\sim}{\sim}$ & 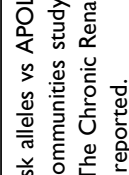 \\
\hline 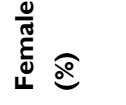 & | & $\stackrel{\infty}{\stackrel{⿲}{\xi}}$ & $\stackrel{m}{q}$ & o̊̀ं & 으 & 0 & రై & q & 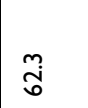 & $\stackrel{\stackrel{O}{\underline{I}}}{ }$ & $\begin{array}{l}0 \\
\dot{\jmath}\end{array}$ & 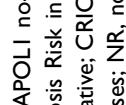 \\
\hline 总 & 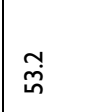 & $\stackrel{\sigma}{\dot{m}}$ & $\dot{H}$ & $\bar{\infty}$ & $\frac{2}{6}$ & $\stackrel{\text { g }}{ }$ & ભે & i̊ & in & กี & nn & 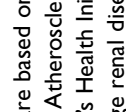 \\
\hline 昱 & $\begin{array}{l}\frac{y}{m} \\
\frac{m}{2} \\
\frac{u}{\alpha} \\
\frac{u}{\alpha}\end{array}$ & 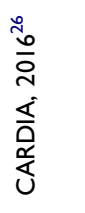 & $\begin{array}{l}\tilde{N}_{m} \\
\bar{\alpha} \\
\dot{y} \\
\frac{\hat{y}}{<}\end{array}$ & $\begin{array}{l}\stackrel{\tilde{m}}{\rho} \\
\bar{i} \\
\frac{u}{\tilde{U}}\end{array}$ & $\begin{array}{l}\pi_{\infty} \\
\frac{\infty}{2} \\
\dot{\overline{1}} \\
\dot{3}\end{array}$ & 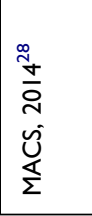 & 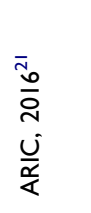 & 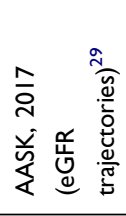 & 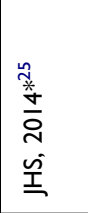 & 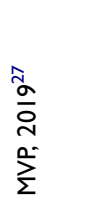 & 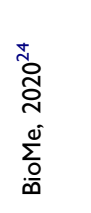 & 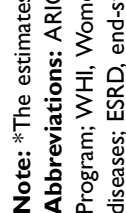 \\
\hline
\end{tabular}


studies were graded as high-quality and three as moderatequality.

\section{APOLI Genotypes and Chronic Kidney Diseases}

Figure 1 summarizes the results of subgroup analyses stratified by the incidence of CKD, ESRD, and CKD to ESRD, respectively. Five studies ${ }^{14,24-27}$ reported data for the association between APOL1 genotype and the risk of CKD. All of the studies reported a positive association (ie, HR $>1.00$ ) between two risk alleles incident CKD compared with zero or one risk allele. High heterogeneity was detected with an $I^{2}=89 \%($ Cochran Q statistic $=36.1, P<0.001)$, the RR from the random-effects model was $1.41(95 \% \mathrm{CI}, 1.14 ; 1.75)$. Progression from CKD to ESRD was available from two studies, ${ }^{23,26}$ from three cohort analyses, with a pooled RR of 1.70 [95CI: 1.44; 2.01] from the fixed-effects model (Figure 1). There was no heterogeneity found with an $I^{2}=0 \%$ (Cochrane Q statistic $=3.0$ ), with all the studies found a RR above 1.00. Incidence of ESRD results was available from 3 studies ${ }^{21,22,27}$ with a pooled RR of 1.86 [95\% CI: 0.90 ; $3.01]$ from the random-effect model. High heterogeneity was found with an $I^{2}=93.2 \%$ (Cochrane Q statistic=29.27, $P<0.0001)$. Two of the studies found a RR above 1.00 except for one study with a non-significant association. ${ }^{24}$ The difference in the risk of APOL1 genetic risk variants with baseline kidney function status was not significant $(\mathrm{P}=0.8414)$. The diagnostic criteria for $\mathrm{CKD} / \mathrm{ESRD}$ incidence varied widely in the included studies (Supplemental Table 1). The Egger regression test did not show statistically significant asymmetry of the funnel plot, suggesting that publication bias was unlikely $(\mathrm{P}=0.1433$; Figure 2).

\section{APOLI Genotypes and Kidney Function Change}

Six studies ${ }^{14,21,23,28,29}$ in 7142 individuals provided data for the association between APOL1 genetic variants and longitudinal eGFR decline rates (Figure 3). The pooled analysis

\section{Author Year}

\section{CKD}

Paranjpe I, 2020 (BioMe)

Bick AG, 2019 (MVP)

Foster MC, 2013 (ARIC)

Ito $\mathrm{K}, 2014$ (JHS)

Peralta CA, 2016 (CARDIA)

Overall effect

Heterogeneity: $l^{2}=89 \%, p<0.01$

\section{CKD to ESRD}

Parsa A, 2013 (CRIC:DM)

Parsa A, 2013 (CRIC:non-DM)

Parsa A, 2013 (AASK)

Foster MC, 2013 (ARIC)

Overall effect

Heterogeneity: $I^{2}=0 \%, p=0.51$

\section{ESRD}

Franceschini N, 2018 (WHI)

Grams ME, 2016 (ARIC)

Bick AG, 2019 (MVP)

Overall effect

Heterogeneity: $l^{2}=93 \%, p<0.01$

\section{Overall effect}

Heterogeneity: $l^{2}=95 \%, p<0.01$

Residual heterogeneity: $I^{2}=87 \%, p<0.01$

\section{Effect Size Standard error}

$\begin{array}{ll}0.02 & 0.0597 \\ 0.17 & 0.0195 \\ 0.41 & 0.2032 \\ 0.70 & 0.1107 \\ 0.70 & 0.2258\end{array}$

$\begin{array}{ll} & \\ & \\ 0.38 & 0.1546 \\ 0.48 & 0.1892 \\ 0.63 & 0.1279 \\ 0.89 & 0.4549\end{array}$

RR $\quad 95 \%-\mathrm{Cl}$ Weight

$1.02[0.91 ; 1.15] \quad 9.9 \%$

$1.18[1.14 ; 1.23] \quad 10.1 \%$

$1.51[1.01 ; 2.25] \quad 7.7 \%$

$2.01[1.62 ; 2.50] \quad 9.3 \%$

$2.02[1.30 ; 3.14] \quad 7.3 \%$

$1.41[1.14 ; 1.75] \quad 44.3 \%$

$1.46[1.08 ; 1.98] \quad 8.6 \%$

$1.61[1.11 ; 2.33] \quad 8.0 \%$

$1.88[1.46 ; 2.42] \quad 9.0 \%$

$2.43[1.00 ; 5.93] \quad 4.0 \%$

$1.70[1.44 ; 2.01] 29.6 \%$

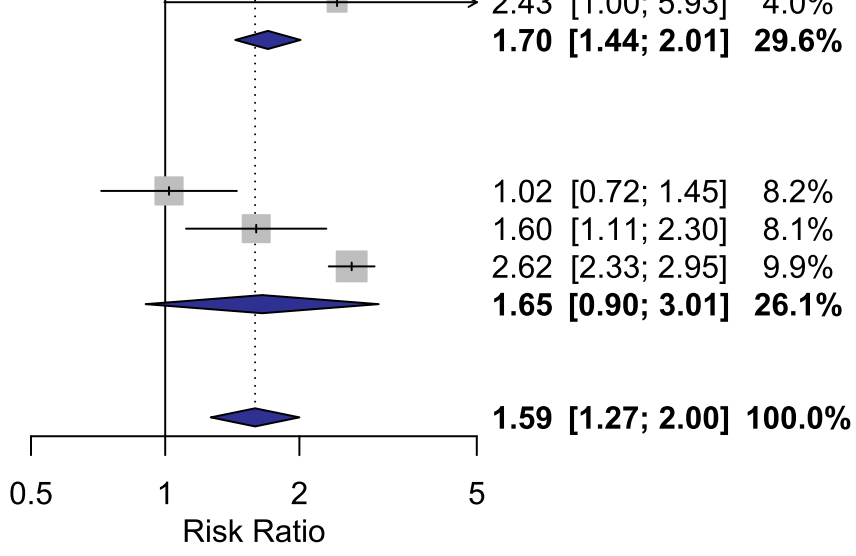

Figure I Association between APOLI high-risk variants and kidney diseases.

Note: The estimates are based on APOLI no-risk alleles vs APOLI high-risk alleles.

Abbreviations: ARIC, Atherosclerosis Risk in Communities study; CARDIA, The Coronary Artery Risk Development in Young Adults; AASK, African American Study of Kidney Disease and Hypertension; MVP, Million Veterans Program; CRIC, The Chronic Renal Insufficiency Cohort; WHI, Women's Health Initiative; APOLI LR, APOLI lowrisk variants; APOLI HR, APOLI high-risk variants; CKD, chronic kidney diseases; ESRD, end-stage renal diseases. 


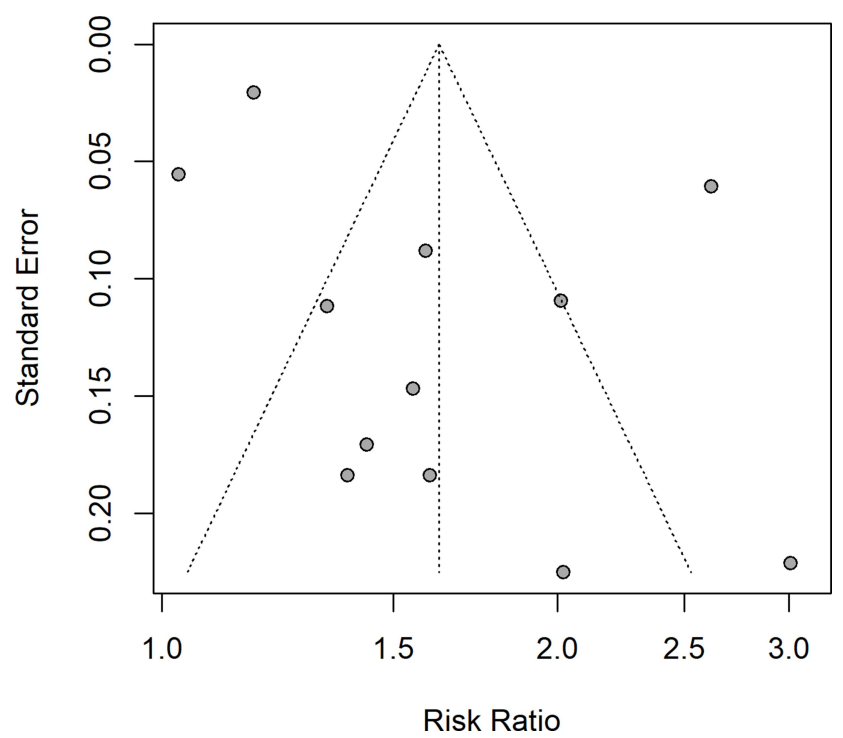

Figure 2 Funnel plot - APOLI and kidney disease events.

showed that the presence of high-risk APOL1 genotype was associated with an annual eGFR decline $(-0.55$ (95\% CI: -0.94 to -0.16$)$ ) $\mathrm{mL} / \mathrm{min} / 1.73 \mathrm{~m}^{2}$ compared with low-risk APOL1 genotype status. There was no significant heterogeneity $\left(\mathrm{I}^{2}=42.0 \% ; \mathrm{P}=0.12\right)$ and publication bias (Egger regression test: $\mathrm{P}=0.2397)$. The high-risk APOL1 genotype mediated decline in kidney function was more pronounced in individuals with CKD $(-0.87[-1.81 ;-0.06] \mathrm{mL} / \mathrm{min} /$ $\left.1.73 \mathrm{~m}^{2}\right)$ at baseline compared without CKD $(-0.27[-0.58$; $0.03] \mathrm{mL} / \mathrm{min} / 1.73 \mathrm{~m}^{2}$ ). In a sensitivity analysis, excluding an HIV cohort (MACS) did not modify the results.

\section{Discussion}

Our meta-analysis of published prospective studies found that high-risk APOL1 genotype status was associated with $\sim 41 \%$ increased risk of CKD and 70\% increased risk of CKD to ESRD progression compared to low-risk APOL1 genotype status. There was no significant association between highrisk APOL1 genotype status with the incidence of ESRD. Our systematic review and meta-analysis are timely, considering recent interests and suggestions regarding APOL1 genetic testing as part of clinical practice or population screening and consideration of the APOL1 genetic testing and treatment in precision medicine. ${ }^{6}$ We found significantly increased variation in study findings than would be anticipated as a result of chance, owing to the considerable heterogeneities in study populations and methods.

Congruently, our study found that high-risk APOL1 genotype status was associated with only a modest reduction in eGFR compared with low-risk APOL1 status. We found increased variability in the annual eGFR decline between the studies. These differences may be due to differences in study design and follow-up duration: for example CRIC participants (with all the participants have CKD at baseline) were followed for a mean of $\sim 4.2$ years, whereas ARIC participants (general population with a normal kidney function) had up to $\sim 25$ years of follow-up.

The plausible biological mechanisms by which APOL1 variants cause kidney failure are not completely understood. Several mechanisms of APOL1 risk variantinduced cell injury have been proposed, such as loss of intracellular $\mathrm{K}^{+}$influx and aberrant activation of stressactivated protein kinase (SAPK) signaling, ${ }^{30}$ mitochondrial and endosomal trafficking dysfunction, ${ }^{31,32}$ and activation of protein kinase $\mathrm{R}$ signaling pathway by increased expression of APOL1 risk allele RNA. ${ }^{33}$

Overall, the pooled analysis of our meta-analysis showed that $\sim 8.5$ million African Americans (16.5\%)
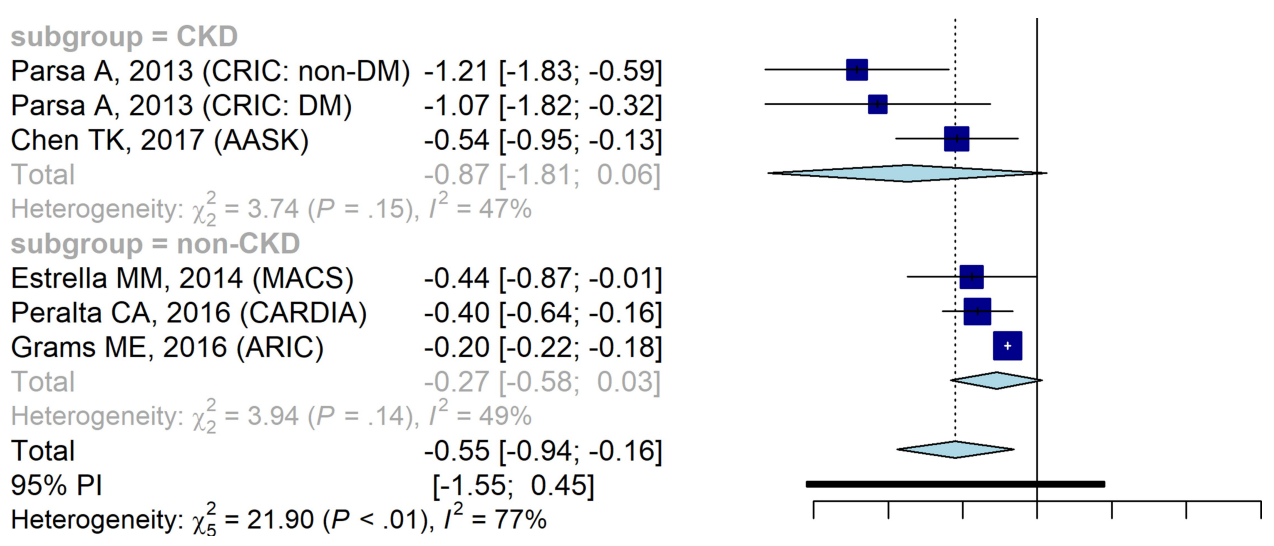

Figure 3 Forest plot showing the annual differences in eGFR decline by APOLI genotype (APOLI high-risk vs APOLI low-risk status). Abbreviations: ARIC, Atherosclerosis Risk in Communities study; CARDIA, The Coronary Artery Risk Development in Young Adults; AASK, African American Study of Kidney Disease and Hypertension; CRIC, The Chronic Renal Insufficiency Cohort; CKD, chronic kidney diseases; ESRD, end-stage renal diseases. 
African Americans potentially carry high-risk APOL1 genotype status, which suggests that APOL1 carries a significant public health burden in the US. However, as shown in our meta-analysis, only a subset of individuals with the high-risk APOL1 genotype progress to CKD. This should prompt the development of studies aiming to identify the biological mechanisms of APOL1 associated nephropathy and identify putative environmental and biomarkers "second hits" to address the growing epidemic of kidney diseases in African Americans. So far, we have not yet determined which factors heighten the disease risk. Identifying subgroups of patients that may benefit from APOL1 screening and culturally-appropriate interventions through the implementation of preventive measures and maybe one day through the development of specific drugs targeting the pathway linking APOL1 and CKD development and progression should be a research priority. ${ }^{6}$

This systematic review and meta-analysis have some limitations. First, the ascertainment of CKD and ESRD outcomes by non-uniform methodologies may provide heterogeneous results, as different methods have various sensitivities and specificities to $\mathrm{CKD} / \mathrm{ESRD}$ diagnosis. Second, we did not identify any prospective studies available on other forms of APOL1 associated nephropathies, such as HIV-associated nephropathy, due to our stringent inclusion criteria. Finally, the available future data were exclusively from the North American African American population and did not include other African regions, as the available studies were of case-control or cross-sectional study design. ${ }^{34-36}$ Therefore, the results are not generalizable globally.

To summarize, our study demonstrates that there was a modest association between high-risk APOL1 genotype with progression to CKD and from CKD to ESRD progression. Currently, there is not sufficient straightforward evidence for guidance, and it may be prudent to conduct APOL1 genotype testing only in high-risk individuals such as in patients with the presence of CKD or HIV who wish to pursue genetic testing after being informed of all advantages and trade-off that are associated with it.

\section{Disclosure}

Dr Allyson Hart reports grants from CSL Behring, outside the submitted work. The authors report no other conflicts of interest in this work.

\section{References}

1. Norton JM, Moxey-Mims MM, Eggers PW, et al. Social determinants of racial disparities in CKD. J Am Soc Nephrol. 2016;27:2576-2595. doi:10.1681/ASN.2016010027

2. Derose SF, Rutkowski MP, Crooks PW, et al. Racial differences in estimated GFR decline, ESRD, and mortality in an integrated health system. Am J Kidney Dis. 2013;62:236-244. doi:10.1053/j.ajkd.20 13.01.019

3. Dummer PD, Limou S, Rosenberg AZ, et al. APOL1 kidney disease risk variants: an evolving landscape. Semin Nephrol. 2015;35:22 2-236. doi:10.1016/j.semnephrol.2015.04.008

4. Tzur S, Rosset S, Shemer R, et al. Missense mutations in the APOL1 gene are highly associated with end stage kidney disease risk previously attributed to the MYH9 gene. Hum Genet. 2010;12 8:345-350. doi:10.1007/s00439-010-0861-0

5. Langefeld CD, Comeau ME, Ng MCY, et al. Genome-wide association studies suggest that APOL1-environment interactions more likely trigger kidney disease in African Americans with nondiabetic nephropathy than strong APOL1-second gene interactions. Kidney Int. 2018;94:599-607. doi:10.1016/j.kint.2018.03.017

6. Kopp JB, Winkler CA. Genetic testing for APOL1 genetic variants in clinical practice: finally starting to arrive. CJASN. 2020;15:126-128. doi: $10.2215 /$ CJN.01810219

7. Young BA, Fullerton SM, Wilson JG, et al. Clinical genetic testing for APOL1: are we there yet? Semin Nephrol. 2017;37:552-557. doi:10.1016/j.semnephrol.2017.07.009

8. Stroup DF, Berlin JA, Morton SC, et al. Meta-analysis of observational studies in epidemiology: a proposal for reporting. Meta-analysis Of Observational Studies in Epidemiology (MOOSE) group. JAMA. 2000;283:2008-2012. doi:10.1001/ jama.283.15.2008

9. Wallace BC, Small K, Brodley CE, Lau J, Trikalinos TA. Deploying an interactive machine learning system in an evidence-based practice center: abstrackr. Paper presented at: Proceedings of the 2nd ACM SIGHIT International Health Informatics Symposium; 2012; Miami, Florida, USA.

10. Stang A. Critical evaluation of the Newcastle-Ottawa scale for the assessment of the quality of nonrandomized studies in meta-analyses. Eur J Epidemiol. 2010;25:603-605. doi:10.1007/ s10654-010-9491-z

11. Higgins JPT, Altman DG, Gøtzsche PC, et al. The Cochrane Collaboration's tool for assessing risk of bias in randomised trials. BMJ. 2011;343:d5928-d5928. doi:10.1136/bmj.d5928

12. Genovese G, Friedman DJ, Ross MD, et al. Association of trypanolytic ApoL1 variants with kidney disease in African Americans. Science. 2010;329:841-845. doi:10.1126/science.1193032

13. Nurminen M. To use or not to use the odds ratio in epidemiologic analyses? Eur J Epidemiol. 1995;11:365-371. doi:10.1007/BF0172 1219

14. Peralta CA, Bibbins-Domingo K, Vittinghoff E, et al. APOL1 genotype and race differences in incident albuminuria and renal function decline. J Am Soc Nephrol. 2016;27:887-893. doi:10.1681/ASN.20 15020124

15. Zhang J, Yu KF. What's the relative risk? A method of correcting the odds ratio in cohort studies of common outcomes. JAMA. 1998;280:1690-1691. doi:10.1001/jama.280.19.1690

16. DerSimonian R, Laird N. Meta-analysis in clinical trials. Control Clin Trials. 1986;7:177-188. doi:10.1016/0197-2456(86)90046-2

17. Schwarzer G,Package 'meta'. General Package for Meta-Analysis. Available from: https://cran.r-project.org/web/packages/meta/meta. pdf. Accessed March 11, 2021.

18. Darroch JN. The Mantel-Haenszel test and tests of marginal symmetry; fixed-effects and mixed models for a categorical response, correspondent paper. Int Stat Rev Int De Stat. 1981;285-307. 
19. Sterne JA, Gavaghan D, Egger M. Publication and related bias in meta-analysis: power of statistical tests and prevalence in the literature. J Clin Epidemiol. 2000;53:1119-1129. doi:10.1016/S089 5-4356(00)00242-0

20. Duval S, Tweedie R. Trim and fill: a simple funnel-plot-based method of testing and adjusting for publication bias in meta-analysis. Biometrics. 2000;56:455-463. doi:10.1111/j.0006-34 1X.2000.00455.X

21. Grams ME, Rebholz CM, Chen Y, et al. Race, APOL1 risk, and eGFR decline in the general population. $J$ Am Soc Nephrol. 2016;27:2842-2850. doi:10.1681/ASN.2015070763

22. Franceschini N, Kopp JB, Barac A, et al. Association of APOL1 with heart failure with preserved ejection fraction in postmenopausal African American Women. JAMA Cardiol. 2018;3:712-720. doi:10. 1001/jamacardio.2018.1827

23. Parsa A, Kao WH, Xie D, et al. Investigators AS and Investigators CS. APOL1 risk variants, race, and progression of chronic kidney disease. N Engl J Med. 2013;369:2183-2196. doi:10.1056/NEJMoa 1310345

24. Paranjpe I, Chaudhary K, Paranjpe M, et al. Association of APOL1 risk genotype and air pollution for kidney disease. CJASN. 2020;15:401-403. doi:10.2215/CJN.11921019

25. Ito K, Bick AG, Flannick J, et al. Increased burden of cardiovascular disease in carriers of APOL1 genetic variants. Circ Res. 2014;114:845-850. doi:10.1161/CIRCRESAHA.114.302347

26. Foster MC, Coresh J, Fornage M, et al. APOL1 variants associate with increased risk of CKD among African Americans. J Am Soc Nephrol. 2013;24:1484-1491. doi:10.1681/ASN.2013010113

27. Bick AG, Akwo E, Robinson-Cohen C, et al. Association of APOL1 risk alleles with cardiovascular disease in blacks in the million veteran program. Circulation. 2019;140:1031-1040. doi:10.1161/ CIRCULATIONAHA.118.036589

28. Estrella MM, Li M, Tin A, et al. The association between APOL1 risk alleles and longitudinal kidney function differs by HIV viral suppression status. Clin Infect Dis. 2015;60:646-652. doi:10.1093/ $\mathrm{cid} / \mathrm{ciu} 765$
29. Chen TK, Appel LJ, Grams ME, et al. APOL1 risk variants and cardiovascular disease: results from the AASK (African American Study of Kidney Disease and Hypertension). Arterioscler Thromb Vasc Biol. 2017;37:1765-1769. doi:10.1161/ATVBAHA.117.309384

30. Olabisi OA, Zhang J-Y, VerPlank L, et al. APOL1 kidney disease risk variants cause cytotoxicity by depleting cellular potassium and inducing stress-activated protein kinases. Proc Natl Acad Sci USA. 2016;113:830-837. doi:10.1073/pnas.1522913113

31. Ma L, Chou JW, Snipes JA, et al. APOL1 renal-risk variants induce mitochondrial dysfunction. J Am Soc Nephrol. 2017;28:1093-1105. doi:10.1681/ASN.2016050567

32. Kruzel-Davila E, Shemer R, Ofir A, et al. APOL1-mediated cell injury involves disruption of conserved trafficking processes. JASN. 2017;28:1117-1130. doi:10.1681/ASN.2016050546

33. Okamoto K, Rausch JW, Wakashin H, et al. APOL1 risk allele RNA contributes to renal toxicity by activating protein kinase R. Commun Biol. 2018;1:188. doi:10.1038/s42003-018-0188-2

34. Kasembeli AN, Duarte R, Ramsay M, et al. APOL1 risk variants are strongly associated with HIV-associated nephropathy in Black South Africans. J Am Soc Nephrol. 2015;26:2882-2890. doi:10.1681/ASN. 2014050469

35. Tayo BO, Kramer H, Salako BL, et al. Genetic variation in APOL1 and MYH9 genes is associated with chronic kidney disease among Nigerians. Int Urol Nephrol. 2013;45:485-494. doi:10.1007/s11255012-0263-4

36. Ulasi II, Tzur S, Wasser WG, et al. High population frequencies of APOL1 risk variants are associated with increased prevalence of non-diabetic chronic kidney disease in the Igbo people from south-eastern Nigeria. Nephron Clin Pract. 2013;123:123-128. doi:10.1159/000353223
The International Journal of Nephrology and Renovascular Disease is an international, peer-reviewed open-access journal focusing on the pathophysiology of the kidney and vascular supply. Epidemiology, screening, diagnosis, and treatment interventions are covered as well as basic science, biochemical and immunological studies. The manuscript management system is completely online and includes a very quick and fair peer-review system, which is all easy to use. Visit http://www.dovepress.com/testimonials.php to read real quotes from published authors. 